\title{
Homotopy methods for constraint relaxation in unilevel reliability based design optimization
}

\author{
Harish Agarwal* Shawn E. Gano ${ }^{\dagger}$ John E. Renaud ${ }^{\ddagger}$ \\ University of Notre Dame, Notre Dame, IN 46556 \\ Victor M. Perez ${ }^{\S}$ \\ General Electric Company, Quertaro, Mexico \\ Layne T. Watson ब \\ Virginia Polytechnic Institute and State University, Blacksburg, VA 24061-0106
}

Reliability based design optimization is a methodology for finding optimized designs that are characterized with a low probability of failure. The main objective in reliability based design optimization is to minimize a merit function while satisfying the reliability constraints. The reliability constraints are constraints on the probability of failure corresponding to each of the failure modes of the system or a single constraint on the system probability of failure. The probability of failure is usually estimated by performing a reliability analysis. During the last few years, a variety of different techniques have been developed for reliability based design optimization. Traditionally, these have been formulated as a double-loop (nested) optimization problem. The upper level optimization loop generally involves optimizing a merit function subject to reliability constraints and the lower level optimization loop(s) compute the probabilities of failure corresponding to the failure mode(s) that govern the system failure. This formulation is, by nature, computationally intensive. A new efficient unilevel formulation for reliability based design optimization was developed by the authors in earlier studies. In this formulation, the lower level optimization (evaluation of reliability constraints in the double loop formulation) was replaced by

\footnotetext{
*Graduate Research Assistant, Aerospace and Mechanical Engineering, email: Agarwal.6@nd.edu.

${ }^{\dagger}$ Graduate Research Assistant, Aerospace and Mechanical Engineering, email: sgano@nd.edu.

¥Professor, Aerospace and Mechanical Engineering, email:jrenaud@nd.edu.

$\S$ GE-CIAT, Queretaro, Mexico. email: victor.perez@ae.ge.com.

ๆProfessor, Departments of Computer Science and Mathematics. email: ltw@cs.vt.edu
} 
its corresponding first order Karush-Kuhn-Tucker (KKT) necessary optimality conditions at the upper level optimization. It was shown that the unilevel formulation is computationally equivalent to solving the original nested optimization if the lower level optimization is solved by numerically satisfying the KKT conditions (which is typically the case), and the two formulations are mathematically equivalent under constraint qualification and generalized convexity assumptions. In the unilevel formulation, the KKT conditions of the inner optimization for each probabilistic constraint evaluation are imposed at the system level as equality constraints. Most commercial optimizers are usually numerically unreliable when applied to problems accompanied by many equality constraints. In this investigation an optimization framework for reliability based design using the unilevel formulation is developed. Homotopy methods are used for constraint relaxation and to obtain a relaxed feasible design. A series of optimization problems are solved as the relaxed optimization problem is transformed via a homotopy to the original problem. A heuristic scheme is employed in this paper to update the homotopy parameter. The proposed algorithm is illustrated with example problems.

\title{
Nomenclature
}

\author{
d Design Variables \\ p Parameters in Deterministic Optimization \\ X Random Variables \\ U Standard Normal Random Variables \\ $\boldsymbol{\theta}$ Distribution Parameters \\ $\boldsymbol{\eta} \quad$ Limit State Parameters \\ $\mathbf{g}^{R} \quad$ Failure driven or Probabilistic Hard Constraints \\ $\mathbf{g}^{D} \quad$ Deterministic Constraints \\ y Deterministic State Variables \\ Y Random State Variables \\ $\beta_{i} \quad$ Reliability Index of $i^{\text {th }}$ Failure Mode \\ $\mathbf{x}^{*} \quad$ Most Probable Point in $\mathbf{x}$-space \\ $\mathbf{u}^{*} \quad$ Most Probable Point in $\mathbf{u}$-space \\ $f \quad$ Merit Function \\ $\mathbf{g}^{r c} \quad$ Reliability Constraints \\ $P_{i} \quad$ Probability of Failure of $i^{\text {th }}$ Failure Mode
}


$P_{\text {allow }}$ Allowable Probability of Failure for $i^{\text {th }}$ Failure Mode

$P_{\text {sys }} \quad$ System Probability of Failure

$\mathbf{d}^{l} \quad$ Lower Bounds on Design Variables

$\mathbf{d}^{u} \quad$ Upper Bounds on Design Variables

$N_{\text {hard }}$ Number of hard constraints

$N_{\text {soft }} \quad$ Number of soft constraints

$\beta_{\text {reqdi }}$ Desired Reliability Index of $i^{\text {th }}$ Failure Mode

\section{Introduction}

In a deterministic design optimization, designs are often driven to the limit of the design constraints, leaving little or no latitude for uncertainties. The resulting deterministic optimum is usually associated with a high probability of failure of the artifact being designed, due to the influence of uncertainties inherently present during the modeling and manufacturing phases of the artifact and due to uncertainties in the external operating conditions of the artifact. The uncertainties include variations in certain parameters, which are either controllable (e.g., dimensions) or uncontrollable (e.g., material properties), and model uncertainties and errors associated with the simulation tools used for simulation based design ${ }^{12}$ In this research, variational uncertainties are modeled as continuous random variables. Other forms of uncertainty, such as model uncertainties and errors associated with simulation tools, are assumed to be minimal and that the analysis tools can reasonably predict the actual performance behavior.

Optimized deterministic designs determined without considering uncertainties can be unreliable and might lead to catastrophic failure of the artifact being designed. Uncertainties in simulation based design are inherently present and need to be accounted for in the design optimization process. Reliability based design optimization (RBDO) is a methodology that addresses this problem. In designing artifacts with multiple failure modes, it is important that an artifact be designed such that it is sufficiently reliable with respect to each of the critical failure modes or to the overall system failure. The reliability index, or the probability of failure corresponding to either a failure mode or the system, can be computed by performing a reliability analysis. In a RBDO formulation, the critical failure modes in deterministic optimization are replaced with constraints on probabilities of failure corresponding to each of the failure driven modes or with a single constraint on the system probability of failure ${ }^{3}$

Traditionally, researchers have formulated RBDO as a nested optimization problem (also known as a double-loop method). Such a formulation is, by nature, computationally expensive because of the inherent computational expense required for the reliability analysis, which itself involves the solution to an opti- 
mization problem. Solving such nested optimization problems are cost prohibitive, especially for large scale multidisciplinary systems, which are themselves computationally intensive. Moreover, the computational cost associated with RBDO grows exponentially as the number of random variables and the number of critical failure modes increase. To alleviate the high computational cost, researchers have developed sequential RBDO methods. In these methods, a deterministic optimization and a reliability analysis are decoupled, and the procedure is repeated until convergence is achieved. However, such techniques usually lead to premature convergence and hence yield spurious optimal designs.

Previous unilevel formulations include those of Liang et al ${ }^{4}$ and the authors, $\frac{[56}{6}$ all using KKT conditions for the lower level problems at the upper level, but differing in the details of the lower level reliability metric, how the KKT conditions are used, or the choice of variables. All these unilevel formulations provided improved computational robustness and provable convergence. The basic idea here is to replace the inverse FORM problem (lower level optimization in the performance measure approach (PMA)) by its first order KKT necessary optimality conditions at the upper level optimization. This unilevel method was shown to be computationally equivalent to the original nested optimization problem if the lower level optimization problem is solved by satisfying the (necessary under a constraint qualification, and sufficient under weak generalized convexity assumptions ${ }^{7}$ ) KKT conditions (which is what most numerical optimization algorithms actually do). Thus the unilevel and double-loop formulations are mathematically equivalent under certain conditions but always computationally equivalent in practice.

The unilevel formulation for RBDO is usually accompanied by a large number of equality constraints that often cause numerical difficulty for many commercial optimizers. This is well documented in the numerical analysis literature $\frac{89}{9}$ where homotopy methods have been proposed as a globally convergent technique to solve large difficult nonlinear systems of equations. The success of homotopy algorithms in solving nonlinear equality constraints for which other standard methods fail is also well documented $[8$ This homotopy reformulation of the KKT equality constraints in the unilevel formulation for RBDO is a significant change, since it completely changes how one solves the upper level problem. In this investigation, an optimization algorithm is developed for reliability based design using the unilevel formulation. Homotopy techniques are employed to relax the constraints and to obtain a relaxed feasible design. A series of less difficult optimization problems are solved as the relaxed problem is transformed into the original problem. A heuristic scheme is employed in this paper to update the homotopy parameter. The proposed algorithm is illustrated with example problems. 


\section{Deterministic Design Optimization}

A deterministic design optimization problem can be formulated as

$$
\begin{array}{cll}
\operatorname{minimize} & f(\mathbf{d}, \mathbf{p}) \\
\text { subject to } & g_{i}^{R}(\mathbf{d}, \mathbf{p}) \geq 0 & i=1, \ldots, N_{\text {hard }}, \\
& g_{j}^{D}(\mathbf{d}, \mathbf{p}) \geq 0 & j=1, \ldots, N_{\text {soft }}, \\
& \mathbf{d}^{l} \leq \mathbf{d} \leq \mathbf{d}^{u},
\end{array}
$$

where $\mathbf{d}$ are the design variables and $\mathbf{p}$ are the fixed parameters of the optimization problem. $g_{i}^{R}$ is the $i^{t h}$ hard constraint that models the $i^{\text {th }}$ critical failure mechanism of the system (e.g., stress, deflection,

loads, etc.). $g_{j}^{D}$ is the $j^{t h}$ soft constraint that models the $j^{\text {th }}$ deterministic constraint due to other design considerations (e.g., cost, marketing, etc). The design space is bounded by $\mathbf{d}^{l}$ and $\mathbf{d}^{u}$.

A deterministic optimization formulation does not account for the uncertainties in the design variables and parameters. Optimized designs based on a deterministic formulation are usually associated with a high probability of failure because of the likely violation of certain hard constraints in service. This is particularly true if the hard constraints are active at the deterministic optimum solution. In today's competitive marketplace, it is very important that the resulting designs are optimal as well as reliable. This is usually achieved by replacing a deterministic optimization formulation with a reliability based design optimization formulation, where the critical hard constraints are replaced with reliability constraints.

\section{Reliability Based Design Optimization}

In the last two decades, researchers have proposed a variety of frameworks for efficiently performing reliability based design optimization. A careful survey of the literature reveals that the various RBDO methods can be divided into three broad categories.

\section{A. Double Loop Methods for RBDO}

Traditionally, the reliability based optimization problem has been formulated as a double loop optimization problem. In a typical RBDO formulation, the critical hard constraints are replaced by reliability constraints, 
as in

$$
\begin{array}{cl}
\text { minimize } & f(\mathbf{d}, \mathbf{p}) \\
\text { subject to } & \mathbf{g}^{r c}(\mathbf{X}, \boldsymbol{\eta}) \geq 0, \\
& g_{j}^{D}(\mathbf{d}, \mathbf{p}) \geq 0 \quad j=1, \ldots, N_{\text {soft }}, \\
& \mathbf{d}^{l} \leq \mathbf{d} \leq \mathbf{d}^{u},
\end{array}
$$

where $\mathbf{g}^{r c}$ are the reliability constraints. They are either constraints on probabilities of failure corresponding to each hard constraint or are a single constraint on the overall system probability of failure. In this investigation, only component failure modes are considered. It should be noted that the reliability constraints depend on the random variables $\mathbf{X}$ and limit state parameters $\boldsymbol{\eta}$. The distribution parameters of the random variables are obtained from the design variables $\mathbf{d}$ and the fixed parameters $\mathbf{p}$ (see the section on reliability analysis below). $\mathbf{g}^{r c}$ can be formulated as

$$
g_{i}^{r c}=P_{\text {allow }_{i}}-P_{i}, \quad i=1, \ldots, N_{\text {hard }}
$$

where $P_{i}$ is the failure probability of the hard constraint $g_{i}^{R}$ at a given design, and $P_{\text {allowi }}$ is the allowable probability of failure for this failure mode. The probability of failure is usually estimated by employing standard reliability techniques. A brief description of standard reliability methods is given in the next section. It has to be noted that the RBDO formulation given above (Equations (5)-(8)) assumes that the violation of soft constraints due to variational uncertainties are permissible and can be traded off for more reliable designs. For practical problems, design robustness represented by the merit function and the soft constraints could be a significant issue, one that would require solution to a hybrid robustness and reliability based design optimization formulation.

\section{B. Reliability Analysis}

Reliability analysis is a tool to compute the reliability index or the probability of failure corresponding to a given failure mode or for the entire system.10 The uncertainties are modeled as continuous random variables, $\mathbf{X}=\left(X_{1}, X_{2}, \ldots, X_{n}\right)^{T}$, with known (or assumed) continuously differentiable distribution functions (CDF), $F_{\mathbf{X}}(\mathbf{x})$. The design variables, $\mathbf{d}$, consist of either distribution parameters $\boldsymbol{\theta}$ of the random variables $\mathbf{X}$, such as means, modes, standard deviations, and coefficients of variation, or deterministic parameters, also called limit state parameters, denoted by $\boldsymbol{\eta}$. The design parameters, $\mathbf{p}$, consist of either the means, the modes, or any first order distribution quantities of certain random variables. Mathematically this can be represented 
by the statements

$$
\begin{aligned}
& {[\mathbf{p}, \mathbf{d}]=[\boldsymbol{\theta}, \boldsymbol{\eta}],} \\
& \mathbf{p} \text { is a subvector of } \boldsymbol{\theta} .
\end{aligned}
$$

Random variables can be consistently denoted as $\mathbf{X}(\boldsymbol{\theta})$, and the $i^{\text {th }}$ failure mode can be denoted as $g_{i}^{R}(\mathbf{X}, \boldsymbol{\eta})$. In the following, $\mathbf{x}$ denotes a realization of the random variables $\mathbf{X}$, and the subscript $i$ is dropped without loss of clarity. Letting $g_{i}^{R}(\mathbf{x}, \boldsymbol{\eta}) \leq 0$ represent the failure domain, and $g^{R}(\mathbf{x}, \boldsymbol{\eta})=0$ be the so-called limit state function, the time-invariant probability of failure for the hard constraint is given by

$$
P(\boldsymbol{\theta}, \boldsymbol{\eta})=\int_{g^{R}(\mathbf{x}, \boldsymbol{\eta}) \leq 0} f_{\mathbf{X}}(\mathbf{x}) d \mathbf{x},
$$

where $f_{\mathbf{X}}(\mathbf{x})$ is the joint probability density (PDF) of $\mathbf{X}$. It is almost impossible to find an analytical expression for the above integral. In standard reliability techniques, a probability distribution transformation $T: \mathbb{R}^{n} \rightarrow \mathbb{R}^{n}$ is usually employed. An arbitrary $n$-dimensional random vector $\mathbf{X}=\left(X_{1}, X_{2}, \ldots, X_{n}\right)^{T}$ is mapped into an independent standard normal vector $\mathbf{U}=\left(U_{1}, U_{2}, \ldots, U_{n}\right)^{T}$. This transformation is known as the Rosenblatt Transformation!11 The standard normal random variables are characterized by a zero mean and unit variance. The limit state function in $\mathbf{U}$-space can be obtained as $g^{R}(\mathbf{x}, \boldsymbol{\eta})=g^{R}\left(T^{-1}(\mathbf{u}), \boldsymbol{\eta}\right)=$ $G^{R}(\mathbf{u}, \boldsymbol{\eta})=0$. The failure domain in $\mathbf{U}$-space is $G^{R}(\mathbf{u}, \boldsymbol{\eta}) \leq 0$. Equation (12) thus transforms to

$$
P(\boldsymbol{\theta}, \boldsymbol{\eta})=\int_{G^{R}(\mathbf{u}, \boldsymbol{\eta}) \leq 0} \phi_{\mathbf{U}}(\mathbf{u}) d \mathbf{u}
$$

where $\phi_{\mathbf{U}}(\mathbf{u})$ is the standard normal density. If the limit state function in $\mathbf{U}$-space is affine, i.e., if $G^{R}(\mathbf{u}, \boldsymbol{\eta})=$ $\boldsymbol{\alpha}^{T} \mathbf{u}+\beta$, then an exact result for the probability of failure is $P_{f}=\Phi\left(-\frac{\beta}{\|\boldsymbol{\alpha}\|}\right)$, where $\Phi(\cdot)$ is the cumulative Gaussian distribution function. If the limit state function is close to being affine, i.e., if $G^{R}(\mathbf{u}, \boldsymbol{\eta}) \approx \boldsymbol{\alpha}^{T} \mathbf{u}+\beta$ with $\beta=-\boldsymbol{\alpha}^{T} \mathbf{u}^{*}$, where $\mathbf{u}^{*}$ is the solution of the following optimization problem,

$$
\begin{array}{cl}
\operatorname{minimize} & \|\mathbf{u}\| \\
\text { subject to } & G^{R}(\mathbf{u}, \boldsymbol{\eta})=0,
\end{array}
$$

then the first order estimate of the probability of failure is $P_{f}=\Phi\left(-\frac{\beta}{\|\boldsymbol{\alpha}\|}\right)$, where $\boldsymbol{\alpha}$ represents a normal to the manifold (15) at the solution point. The solution $\mathbf{u}^{*}$ of the above optimization problem, the so-called design point, $\beta$-point or the most probable point (MPP) of failure, defines the reliability index $\beta_{p}=-\frac{\boldsymbol{\alpha}^{T} \mathbf{u}^{*}}{\|\boldsymbol{\alpha}\|}$. This method of estimating the probability of failure is known as the first order reliability method (FORM)! 
In the second order reliability method (SORM), the limit state function is approximated as a quadratic surface. A simple closed form solution for the probability computation using a second order approximation exist ${ }^{[12}$ The solution uses the theory of asymptotic approximations. It is given as

$$
\begin{aligned}
P_{f}(\boldsymbol{\theta}, \boldsymbol{\eta}) & =\int_{G^{R}(\mathbf{u}, \boldsymbol{\eta}) \leq 0} \phi_{\mathbf{U}}(\mathbf{u}) d \mathbf{u} \\
& \approx \Phi\left(-\beta_{p}\right) \prod_{l=1}^{n-1}\left(1-\kappa_{l}\right)^{-1 / 2}
\end{aligned}
$$

where the $\kappa_{l}$ are related to the principal curvatures of the limit state function at the minimum distance point $\mathbf{u}^{*}$, and $\beta_{p}$ is the reliability index using FORM. It was shown that the second order probability estimate asymptotically approaches the first order estimate as $\beta_{p}$ approaches infinity if $\beta_{p} \kappa_{l}$ remains constant.12]

The first order approximation, $P_{f} \approx \Phi\left(-\beta_{p}\right)$, is sufficiently accurate for most practical cases. Thus, only first order approximations of the probability of failure are used in practice. Using the FORM estimate, the reliability constraints in Equation (9) can be written in terms of reliability indices as

$$
g_{i}^{r c}=\beta_{i}-\beta_{r_{e q d}}
$$

where $\beta_{i}$ is the first order reliability index, and $\beta_{\text {reqd }_{i}}=-\Phi^{-1}\left(P_{\text {allow }}\right)$ is the desired reliability index for the $i^{t h}$ hard constraint. When the reliability constraints are formulated as given in equation (17), the approach is referred to as the reliability index approach (RIA).

It should be noted that the reliability analysis involves a probability distribution transformation, the search for the MPP, and the evaluation of the cumulative Gaussian distribution function. To solve the FORM problem (Equations 14 15), various algorithms have been reported in the literature!13 The solution typically requires many function evaluations. Moreover, there might be cases where the optimizer may fail to provide a solution to the FORM problem, especially when the limit state surface is far away from the origin in $\mathbf{U}$-space or when the case $G^{R}(\mathbf{u}, \boldsymbol{\eta})=0$ never occurs at a particular design setting.

In design automation it cannot be known a priori what design points the upper level optimizer will visit, therefore it is not known if the optimizer for the FORM problem will provide a consistent result. This problem was addressed recently by using a trust region algorithm for equality constrained problems 14 For cases when $G^{R}(\mathbf{u}, \boldsymbol{\eta})=0$ does not occur, the algorithm provided the best possible solution for the problem through

$$
\begin{aligned}
\text { minimize } & \|u\| \\
\text { subject to } & G^{R}(\mathbf{u}, \boldsymbol{\eta})=c .
\end{aligned}
$$


The reliability constraints formulated by the RIA are therefore not robust. RIA is usually more effective if the probabilistic constraint is violated, but it yields a singularity if the design has zero failure probability 15 To overcome this difficulty, an improved formulation was developed to solve the RBDO problem.[15] In this method, known as the performance measure approach (PMA), the reliability constraints are stated by an inverse formulation as

$$
g_{i}^{r c}=G_{i}^{R}\left(\mathbf{u}_{\mathbf{i} \beta=\rho}^{*}, \boldsymbol{\eta}\right) \quad i=1, \ldots, N_{\text {hard }} .
$$

$\mathbf{u}_{\mathbf{i}}{ }^{*}$ is the solution to the inverse reliability analysis (IRA) optimization problem

$$
\begin{aligned}
\operatorname{minimize} & G_{i}^{R}(\mathbf{u}, \boldsymbol{\eta}) \\
\text { subject to } & \|\mathbf{u}\|=\rho=\beta_{\text {reqd }_{i}},
\end{aligned}
$$

where the optimum solution $\mathbf{u}_{\mathbf{i}}^{*}{ }_{\beta=\rho}^{*}$ corresponds to MPP in IRA of the $i^{\text {th }}$ hard constraint. Solving RBDO by the PMA formulation is usually more efficient and robust than the RIA formulation where the reliability is evaluated directly. The efficiency lies in the fact that the search for the MPP of an inverse reliability problem is easier than the search of the MPP corresponding to an actual reliability. The RIA and the PMA approaches for RBDO are essentially the inverse of one another and would yield the same solution if the constraints are active at the solution. ${ }^{[15}$ If the constraint on the reliability index (as in the RIA formulation) or the constraint on the optimum value of the limit state function (as in the PMA formulation) is not active at the solution, the solutions obtained from the two approaches might differ. Similar RBDO formulations were independently developed by other researchers. ${ }^{16} 17$ 18] In these RBDO formulations, constraint (22) is considered as an inequality constraint $\left(\|\mathbf{u}\| \leq \beta_{\text {reqd }}\right)$, which is a more robust way of handling the constraint on the reliability index. The major difference lies in the fact that in these papers semi-infinite optimization algorithms were employed to solve the RBDO problem. Semi-infinite optimization algorithms solve the inner optimization problem approximately. However, the overall RBDO is still a nested double loop optimization procedure. As mentioned earlier, such formulations are computationally intensive for problems where the function evaluations are expensive. Moreover, the formulation becomes impractical when the number of hard constraints increase, which is often the case in real-life design problems. To alleviate the computational cost associated with the nested formulation, sequential RBDO methods have been developed.

\section{Sequential Methods for RBDO}

Sequential RBDO methods include a variety of different approaches proposed by different researchers. A sequential optimization and reliability assessment methodology has been developed in Chen and Du!19] In this framework, the deterministic optimization and the reliability assessment are decoupled from one another. 
During each cycle, a deterministic optimization problem is solved, followed by reliability assessment and a convergence check. Similar sequential RBDO methodologies have also been developed by other researchers. ${ }^{20}$ This has been generalized for nonnormal distributions in Wang and Kodiyalam.21] In this methodology, the lower level optimization is eliminated, and the MPP of failure corresponding to the probabilistic constraints is estimated implicitly by using a nonlinear transformation based on the direction cosines of the hard constraints at the mean values of the random variables. This methodology is shown to be extremely efficient 202122 However, for highly nonlinear limit state functions, the estimate of the MPP of failure given by the nonlinear transformation might be very different from the actual MPP of failure, and the framework may fail to converge to the true solution. The drawback of sequential RBDO methodologies is that a local optimum cannot be guaranteed; such methodologies can lead to spurious optimal designs. Agarwal and Renaud ${ }^{23}$ developed a decoupled probabilistic design methodology to address this problem.

It has been noted that the traditional reliability based optimization formulation results in a nested optimization problem. Solving such nested optimization problems for a large number of failure driven constraints and/or nondeterministic variables is extremely expensive. Researchers have developed sequential approaches to speed up the optimization process and to obtain a consistent reliability based design. However, these techniques may lead to spurious optimal solutions.

\section{Unilevel Methods for RBDO}

RBDO is typically formulated as a nested optimization problem requiring a large number of function evaluations. The major concern in evaluating reliability constraints is the fact that reliability analysis methods are formulated as optimization problems. ${ }^{[24}$ To overcome this difficulty, a unilevel formulation was developed by Kuschel and Rackwitz: ${ }^{25}$ In their method, the direct FORM problem (lower level optimization-Eqs.

(14) - (15) ) is replaced by the corresponding first order Karush-Kuhn-Tucker (KKT) optimality conditions of the first order reliability problem. As mentioned earlier, the direct FORM problem can be ill-conditioned, and the same may be true for the unilevel formulation given by Kuschel and Rackwitz. ${ }^{25}$ The reason is that the probabilistic hard constraints might have a zero failure probability at a particular design setting, and hence the optimizer might not converge due to the hard constraints (which are posed as equality constraints) not being satisfied. Moreover, the conditions under which such a replacement is equivalent to the original bilevel formulation was not detailed. Similar ideas appeared later in work by Liang and Mourelatos $\stackrel{4}{4}$

A new unilevel formulation, developed by the authors, has been shown to be computationally equivalent to the original bilevel formulation! $[$ The formulation is described here for the sake of completeness. The conditions under which the formulation is computationally equivalent to the traditional bilevel formulation are KKT constraint qualifications and generalized convexity ${ }^{17}$ assumptions. In this formulation, the inverse 
reliability analysis optimization problem is replaced by the corresponding first order necessary Karush-KuhnTucker (KKT) optimality conditions. The KKT conditions for the reliability constraints similar to PMA (Eqns. (21)-(22) ) are used. The treatment of Eqn. (22) is a bit subtle. No simple modification of Eqn. (22) will result in an equality constraint that is both quasiconvex and quasiconcave, which would be required for the sufficiency of the KKT conditions. For necessity of the KKT conditions, observe that $\|\mathbf{u}\|-\rho$ is convex and $\|\mathbf{u}\|-\rho \leq 0$ trivially satisfies Slater's constraint qualification (feasible set has a strictly interior point)! 7 Assume that $G^{R}(\mathbf{u}, \boldsymbol{\eta})$ is pseudoconvex with respect to $\mathbf{u}$ for each fixed $\boldsymbol{\eta}$. Now $G^{R}(\mathbf{u}, \boldsymbol{\eta})$ pseudoconvex and $\|\mathbf{u}\|-\rho$ convex means that the KKT conditions are also sufficient, hence the original and KKT formulation will be equivalent. Therefore, to facilitate development of the unilevel method, the inverse FORM is restated as

$$
\begin{aligned}
\min & G_{i}^{R}(\mathbf{u}, \boldsymbol{\eta}) \\
\text { subject to } & \|\mathbf{u}\| \leq \rho .
\end{aligned}
$$

The Lagrangian corresponding to the optimization problem is

$$
L=G^{R}(\mathbf{u}, \boldsymbol{\eta})+\lambda(\|\mathbf{u}\|-\rho)
$$

where $\lambda$ is the scalar Lagrange multiplier. The first order necessary conditions for the problem are

$$
\begin{aligned}
& \nabla_{\mathbf{u}} G^{R}\left(\mathbf{u}^{*}, \boldsymbol{\eta}\right)+\lambda \nabla_{\mathbf{u}}\left(\left\|\mathbf{u}^{*}\right\|-\rho\right)=0, \\
& \left\|\mathbf{u}^{*}\right\|-\rho \leq 0 \\
& \lambda \geq 0 \\
& \lambda\left(\left\|\mathbf{u}^{*}\right\|-\rho\right)=0
\end{aligned}
$$

where $\mathbf{u}^{*}$ is the solution point $\mathbf{u}_{\beta=\rho}^{*}$ of the inverse reliability optimization problem when $\left\|\mathbf{u}^{*}\right\|=\rho$. $\mathbf{u}^{*}=0$ is a special degenerate case, so assume henceforth that $\mathbf{u}^{*} \neq 0$. From equation (26), we have (assuming $\lambda \neq 0$ )

$$
\mathbf{u}^{*}=-\frac{1}{\lambda}\left\|\mathbf{u}^{*}\right\| \nabla_{\mathbf{u}} G^{R}\left(\mathbf{u}^{*}, \boldsymbol{\eta}\right)
$$

Observe that Eqn. (30) implies

$$
\lambda=\left\|\nabla_{\mathbf{u}} G^{R}\left(\mathbf{u}^{*}, \boldsymbol{\eta}\right)\right\| \geq 0
$$

which is consistent with Eqn. (28) and is valid even if $\lambda=0$. Substituting for $\lambda$ in equation (30) and 
rearranging,

$$
\frac{\nabla_{\mathbf{u}} G^{R}\left(\mathbf{u}^{*}, \boldsymbol{\eta}\right)}{\left\|\nabla_{\mathbf{u}} G^{R}\left(\mathbf{u}^{*}, \boldsymbol{\eta}\right)\right\|}=-\frac{\mathbf{u}^{*}}{\left\|\mathbf{u}^{*}\right\|}
$$

Eqn. (32) says that $\mathbf{u}^{*}$ and $\nabla_{\mathbf{u}} G^{R}\left(\mathbf{u}^{*}, \boldsymbol{\eta}\right)$ point in opposite directions, which is consistent with $\mathbf{u}^{*}$ being the closest point in the manifold $G^{R}(\mathbf{u}, \boldsymbol{\eta})=$ constant to the origin.

Eqn. (32) is true for all $\boldsymbol{\eta}$, if $\mathbf{u}^{*}=\mathbf{u}_{\beta=\rho}^{*}$ is the solution to the inverse reliability optimization problem, because $\rho-\|\mathbf{u}\| \leq 0$ satisfies the reverse convex constraint qualification (the equality constraint (22) is equivalent to the convex constraint (24) and $\rho-\|\mathbf{u}\| \leq 0$, hence constraint qualifications are satisfied and the KKT condition (32) is necessary). In general, without the pseudoconvexity assumption on $G^{R}$, solving equation (32) does not necessarily imply that $\mathbf{u}^{*}$ is the optimal solution to the optimization problem.

It should be noted that the KKT conditions for the direct and inverse FORM problems differ only in terms of what constraints are being presented as equality constraints to the upper level optimizer. When using the KKT conditions of the direct FORM problem in the upper level optimization, the limit state function is presented as an equality constraint and the constraint on the reliability index is an inequality constraint. As mentioned earlier, it is possible to have cases where the limit state function never becomes zero. In other words, it is associated with zero (or one) failure probability. When such a case occurs, the formulation given by Kuschel and Rackwit 25 might fail to yield a solution. In other words, it is numerically unreliable.

In the unilevel formulation developed in Agarwal et. al. ${ }^{6}$ the first order conditions of the inverse FORM problem are used. The corresponding KKT conditions for the inverse reliability problem (Eqns. (23)-(24) ) are

$$
\begin{aligned}
h 1_{i} & \equiv \nabla_{\mathbf{u}} G_{i}^{R}\left(\mathbf{u}_{i}, \boldsymbol{\eta}\right)+\lambda_{i} \frac{\mathbf{u}_{i}}{\left\|\mathbf{u}_{i}\right\|}=0, \\
g 1_{i} & \equiv\left\|\mathbf{u}_{i}\right\|-\beta_{\text {reqd }_{i}} \leq 0, \\
h 2_{i} & \equiv \lambda_{i}\left(\left\|\mathbf{u}_{i}\right\|-\beta_{\text {reqd }_{i}}\right)=0, \\
g 2_{i} & \equiv \lambda_{i} \geq 0 .
\end{aligned}
$$


Using these first order optimality conditions, the unilevel RBDO architecture can be stated as follows

$$
\begin{array}{cl}
\underset{\mathbf{d}_{\text {aug }}}{\min } & f(\mathbf{d}, \mathbf{p}) \\
& \mathbf{d}_{\text {aug }}=\left[\mathbf{d}, \mathbf{u}_{1}, \ldots, \mathbf{u}_{N_{\text {hard }}}, \lambda_{1}, \ldots, \lambda_{N_{\text {hard }}}\right] \\
\text { sub. to } & \mathbf{G}_{i}^{R}(\mathbf{u}, \boldsymbol{\eta}) \geq 0 \quad i=1, \ldots, N_{\text {hard }}, \\
& h 1_{i}=0 \quad i=1, \ldots, N_{\text {hard }}, \\
& h 2_{i}=0 \quad i=1, \ldots, N_{\text {hard }}, \\
& g 1_{i} \leq 0 \quad i=1, \ldots, N_{\text {hard }}, \\
& g 2_{i} \geq 0 \quad i=1, \ldots, N_{\text {hard }}, \\
& g_{j}^{D}(\mathbf{d}, \mathbf{p}) \geq 0 \quad j=1, \ldots, N_{\text {soft }}, \\
& \mathbf{d}^{l} \leq \mathbf{d} \leq \mathbf{d}^{u} .
\end{array}
$$

If $\mathbf{d}^{*}$ is a solution of Eqns. (5)-(8), then there exist $\mathbf{u}_{i}^{*}$ and $\lambda_{i}^{*}$ such that $\left[\mathbf{d}^{*}, \mathbf{u}_{1}^{*}, \ldots, \mathbf{u}_{N_{\text {hard }}^{*}}, \lambda_{1}^{*}, \ldots, \lambda_{N_{\text {hard }}}^{*}\right]$ is a solution of Eqns. (37)-(44). The converse is true, under the mild assumption that all the functions $G_{i}^{R}(\mathbf{u}, \boldsymbol{\eta})$ are pseudoconvex in $\mathbf{u}$ for each fixed $\boldsymbol{\eta}$.

It should be noted that the dimensionality of the problem has increased, as in the unilevel methods given by Kuschel and Rackwit 255 and others. The optimization is performed with respect to the design variables d, the MPPs of failure, and the Lagrange multipliers, simultaneously. At the beginning of the optimization, $\mathbf{u}_{i}$ does not correspond to the true MPP at the design $\mathbf{d}$. The exact MPPs of failure $\mathbf{u}_{\mathbf{i}}{ }^{*}$ and the optimum design $\mathbf{d}^{*}$ are found at convergence.

In the unilevel formulation developed by the authors, the KKT conditions of the inner optimization for each probabilistic constraint evaluation are imposed at the system level as equality constraints. Most commercial optimizers are usually numerically unreliable when applied to problems accompanied by many equality constraints. In this investigation, homotopy methods are used for constraint relaxation and to obtain a simpler problem for which the solution is known. A series of optimization problems are then solved as the relaxed optimization problem is transformed into the original problem. A heuristic scheme suffices in this paper to update the homotopy parameter, although rigorous, sophisticated updating schemes are known and should be used in general.

\section{Proposed Algorithm}

Since the problem of interest is accompanied by a large number of equality constraints, it is extremely important that the constraint relaxation techniques be such that it is easier to identify an initial feasible 
starting point. In this paper, homotopy methods have been used for this purpose $\frac{9]}{9}$ Homotopy techniques have been shown to be extremely robust in the work by Pérez et. al ${ }^{[26}$ The constraint relaxation used in this investigation is of the form

$$
\begin{aligned}
& g r \equiv g+(1-\tau) b \geq 0, \\
& h r \equiv h+(1-\tau) c=0,
\end{aligned}
$$

where $g \geq 0$ and $h=0$ are generic inequality and equality constraints, $g r \geq 0$ and $h r=0$ are the relaxed inequality and equality constraints, respectively. The constants $b$ and $c$ are chosen to make the relaxed constraints feasible at the beginning. For the inequalities, $b$ is based on the value of $g$. If $g \geq 0, b$ is set equal to zero. If $g<0, b$ is set equal to the negative of $g$. Similarly, for the equalities, the constant $c$ is chosen to satisfy the relaxed equality constraint at the initial design. The homotopy parameter $\tau$ drives the relaxed constraints to the original constraints by gradually adjusting $\tau=0 \rightarrow \tau=1$.

After each cycle, the homotopy parameter $\tau$ is updated. In this investigation, it is incremented by a constant value (so the algorithm here is continuation, not a general homotopy algorithm部). Note that varying the homotopy parameter $\tau$ yields a sequence of simpler problems starting from a known solution. When the parameter reaches 1 , the solution to the original problem is found. Conditions under which this process is guaranteed to work are studied in Watson. [9]

\section{Test Problems}

The proposed algorithm is implemented for a short rectangular column design problem and a steel column design problem. Both the test problems are taken from the literature and have been used to test RBDO methodologies.

\section{A. Short Rectangular Column 27}

The design problem is to determine the depth $h$ and width $b$ of a short column with rectangular cross section with a minimal total mass $b h$ assuming unit mass per unit area. The uncertain vector, $\mathbf{X}=(P, M, Y)$, the stochastic parameters, and the correlations of the vector elements are listed in Table 1 The limit state function in terms of the random vector, $\mathbf{X}=(P, M, Y)$, and the limit state parameters, $\boldsymbol{\eta}=(b, h)$, (which happens to be same as the design vector $\mathbf{d}$ in this problem) is given by

$$
g^{R}(\mathbf{X}, \boldsymbol{\eta})=1-\frac{4 M}{b h^{2} Y}-\frac{P^{2}}{(b h Y)^{2}}
$$


The objective function is given by

$$
f(\mathbf{d})=b h .
$$

The depth $h$ and the width $b$ of the rectangular column had to satisfy $15 \leq h \leq 25$ and $5 \leq b \leq 15$. The allowable failure probability is 0.00621 or in other words a reliability index for the failure mode greater than or equal to 2.5. The optimization process was started from the point $\left(\mathbf{u}^{0}, \mathbf{d}^{0}, \lambda^{0}\right)=((1,1,-1),(5,15), 0.1)$. The optimal solution for this problem is $\mathbf{d}^{*}=(8.668,25.0)$.

Figure 1] shows the history of the objective function. It is noted that as the value of the homotopy parameter $\tau$ increases from 0 to 1 , the objective function gradually approaches the optimal solution.

Figure 2 shows the history of the augmented design variables. Observe that the variables gradually approach the optimal solution of the original problem. The homotopy parameter $\tau$ controls the progress of the optimization process. For highly nonlinear problems, it might be difficult to locate the solution directly, or even find a feasible point. The use of the homotopy parameter allows one to start from a known feasible solution and gradually make progress towards the optimal solution.

\section{B. Steel Column 27}

The problem is a steel column with design vector, $\mathbf{d}=(b, d, h)$, where

$b=$ mean of flange breadth,

$d=$ mean of flange thickness, and

$h=$ mean of height of steel profile.

The length of the steel column $(s)$ is $7500 \mathrm{~mm}$. The objective is to minimize the cost function, $f=b d+5 h$. The independent random vector, $\mathbf{X}=\left(F_{s}, P_{1}, P_{2}, P_{3}, B, D, H, F_{0}, E\right)$, and its stochastic characteristics are given in Table 2

The limit state function in terms of the random vector, $\mathbf{X}$, the limit state parameters, $\boldsymbol{\eta}=\mathbf{d}$, is given as

$$
G^{R}(\mathbf{X}, \boldsymbol{\eta})=F_{s}-\mathcal{P}\left(\frac{1}{\mathcal{A}_{s}}+\frac{F_{0}}{\mathcal{M}_{s}} \cdot \frac{\epsilon_{b}}{\epsilon_{b}-\mathcal{P}}\right)
$$

where

$$
\begin{array}{lr}
\mathcal{A}_{s}=2 B D, \quad \text { (area of section) } \\
\mathcal{M}_{s}=B D H, \quad \text { (modulus of section) } \\
\mathcal{M}_{i}=\frac{1}{2} B D H^{2}, & \text { (moment of inertia) } \\
\epsilon_{b}=\frac{\pi^{2} E \mathcal{M}_{i}}{s^{2}}, & \text { (Euler buckling load) }
\end{array}
$$

The means of the flange breadth $b$ and flange thickness $d$ must be within the intervals $[200,400]$ and [10,30], 
respectively. The interval $[100,500]$ defines the admissible mean height $h$ of the T-shaped steel profile. It is required that the optimal design satisfy a reliability level of 3 .

The optimal solution for this problem is $\mathbf{d}=(200,17.1831,100)$. Similar convergence history was observed for this test problem as well. Figure 3 shows the convergence of the objective function. Again it is observed that the homotopy parameter controls the progress of the optimization process toward a solution.

\section{Conclusions}

In this paper, an optimization methodology using homotopy techniques is presented for reliability based design. An efficient and robust unilevel formulation developed earlier by the authors is employed. From the authors' experience, the unilevel formulation for RBDO, when directly coupled with an optimizer, may not converge if there are many equality constraints or if the problem is sufficiently nonlinear. Since the unilevel formulation is usually accompanied by many equality constraints, homotopy techniques are used to relax the constraints and identify a starting point that is feasible with respect to the relaxed constraints. In this investigation, the homotopy parameter is incremented by a fixed value, recognizing that in general a sophisticated homotopy curve tracking algorithm will be needed. A series of optimization problems are solved for increasing values of the homotopy parameter as the relaxed problem approaches the original problem. It is often easier to solve the relaxed problem from a known (relaxed) solution and make gradual progress towards the solution than solve the original problem directly. The proposed strategy is tested on two design problems. It is observed that the homotopy parameter controls the progress made in each cycle of the optimization process. As the homotopy parameter approaches the value of 1, the optimal solution is obtained. Future work includes replacing the naive homotopy parameter incrementation with a true homotopy curve tracking algorithm, and solving a realistic large scale RBDO problem with the proposed technique.

\section{Acknowledgments}

This research was supported in part by the Center for Applied Mathematics at the University of Notre Dame, NSF grants DMI01-1497, DMI-0422719, DMI-0355391, Department of Energy Grant DE-FG0206ER25720, and ONR grant N00014-02-1-0786.

\section{References}

${ }^{1}$ Oberkampf, W. L., DeLand, S. M., Rutherford, B. M., Diegert, K. V., and Alvin, K. F., "Estimation of Total Uncertainty in Modeling and Simulation," Tech. Rep. SAND2000-0824, Sandia National Laboratories, April 2000.

${ }^{2}$ Oberkampf, W. L., Helton, J. C., and Sentz, K., "Mathematical Representation of Uncertainty," Proceedings of the 42nd AIAA/ASME/ASCE/AHS/ASC Structures, Structural Dynamics, and Materials Conference \& Exhibit, No. AIAA 2001-1645, 
Seattle, WA, April 16-19 2001.

${ }^{3}$ Enevoldsen, I. and Sorensen, J. D., "Reliability-based optimization in structural engineering," Structural Safety, Vol. 15, No. 3, 1994, pp. 169-196.

${ }^{4}$ Liang, J., Mourelatos, Z. P., and Tu, J., "A single-loop method for reliability-based design optimization," Proceedings of the ASME Design Engineering Technical Conferences (DETC), 2004.

${ }^{5}$ Agarwal, H., Renaud, J. E., Lee, J. C., and Watson, L. T., "A Unilevel Method For Reliability Based Design Optimization," Proceedings of the 45th AIAA/ASME/ASCE/AHS/ASC Structures, Structural Dynamics, and Materials Conference 8 Exhibit, No. AIAA 2004-2029, Palm Springs, CA, April 19-22 2001.

${ }^{6}$ Agarwal, H., Mozumder, C. K., Renaud, J. E., and Watson, L. T., "An inverse-measure-based unilevel architecture for reliability-based design optimization," Structural and Multidisciplinary Optimization, Vol. 33, 2007, pp. $217-227$.

${ }^{7}$ Mangasarian, O. L., Nonlinear Programming, Classics in Applied Mathematics, SIAM, Philadelphia, 1994.

${ }^{8}$ Watson, L. T., "Engineering applications of the Chow-Yorke algorithm," Applied Mathematics and Computation, Vol. 9, 1981, pp. 111-133.

${ }^{9}$ Watson, L. T., "Theory of globally convergent probability-one homotopies for nonlinear programming," SIAM Journal on Optimization, Vol. 11, No. 3, 2000, pp. 761-780.

${ }^{10}$ Haldar, A. and Mahadevan, S., Probability, Reliability and Statistical Methods in Engineering Design, John Wiley \& Sons, 2000.

${ }^{11}$ Rosenblatt, M., "Remarks on a multivariate transformation," The Annals of Mathematical Statistics, Vol. 23, No. 3, September 1952, pp. 470-472.

${ }^{12}$ Brietung, K., "Asymptotic approximations for multinormal integral," Journal of Engineering Mechanics, Vol. 110, No. 3, 1984, pp. 357-366.

${ }^{13}$ Liu, P.-L. and Kiureghian, A. D., "Optimization algorithms for structural reliability," Structural Safety, Vol. 9, No. 3, 1991, pp. 161-177.

${ }^{14}$ Padmanabhan, D. and Batill, S. M., "Reliability Based Optimization Using Approximations with Applications to MultiDisciplinary System Design," Proceedings of the 40th AIAA Sciences Meeting \& Exhibit, No. AIAA-2002-0449, Reno, NV, January 2002.

${ }^{15} \mathrm{Tu}$, J., Choi, K. K., and Park, Y. H., "A new study on reliability-based design optimization," Journal of Mechanical Design, Vol. 121, December 1999, pp. 557-564.

${ }^{16}$ Polak, E., Wets, R. J.-B., and der Kiureghian, A., "On an approach to optimization problems with a probabilistic cost and or constraints," Nonlinear Optimization and Related Topics, 2000, pp. 299-316.

${ }^{17}$ Royset, J. O., Kiureghian, A. D., and Polak, E., "Reliability based optimal structural design by the decoupling approach," Reliability Engineering and System Safety, Vol. 73, No. 3, 2001, pp. 213-221.

${ }^{18}$ Kirjner-Neto, C., Polak, E., and der Kiureghian, A., "An outer approximations approach to reliability-based optimal design of structures," Journal of Optimization Theory and Applications, Vol. 98, No. 1, July 1998, pp. 1-16.

${ }^{19}$ Chen, W. and Du, X., "Sequential Optimization and Reliability Assesment Method for Efficient Probabilistic Design," ASME Design Engineering Technical Conferences and Computers and Information in Engineering Conference, No. DETC2002/DAC-34127, Montreal, Canada, 2002.

${ }^{20}$ Chen, X. C., Hasselman, T. K., and Neill, D. J., "Reliability Based Structual Design Optimization For Practical Applications," Proceedings of the 38th AIAA/ASME/ASCE/AHS/ASC Structures, Structural Dynamics, and Materials Conference, No. AIAA-97-1403, 1997, pp. 2724-2732.

${ }^{21}$ Wang, L. and Kodiyalam, S., "An Efficient Method For Probabilistic and Robust Design With Non-Normal Distribution," 
Proceedings of the 43rd AIAA/ASME/ASCE/AHS/ASC Structures, Structural Dynamics, and Materials Conference, No. AIAA 2002-1754, Denver, Colorado, April 22-25 2002.

${ }^{22}$ Agarwal, H., Renaud, J. E., and Mack, J. D., "A Decomposition Approach for Reliability-Based Multidisciplinary Design Optimization," Proceedings of the 44th AIAA/ASME/ASCE/AHS/ASC Structures, Structural Dynamics, and Materials Conference ES Exhibit, No. AIAA 2003-1778, Norfolk, Virginia, April 7-10 2003.

${ }^{23}$ Agarwal, H. and Renaud, J. E., "Decoupled methodology for Probabilistic Design Optimization," ASCE Joint Specialty Conference on Probabilistic Mechanics and Structural Reliability, Albuquerque, NM, July 26-28 2004.

${ }^{24}$ Rackwitz, R., "Reliability analysis-a review and some perspectives," Structural Safety, Vol. 23, No. 4, 2001, pp. 365-395.

${ }^{25}$ Kuschel, N. and Rackwitz, R., "A new approach for structural optimization of series systems," Applications of Statistics and Probability, Vol. 2, No. 8, 2000, pp. 987-994.

${ }^{26}$ Pérez, V. M., Renaud, J. E., and Watson, L. T., "Interior Point Sequential Approximate Optimization Methodology," Proceedings of the 10th AIAA/NASA/USAF/ISSMO Symposium on Multidisciplinary Analysis ES Optimization, No. AIAA2002-5505, Atlanta, GA., September 4-6 2002.

${ }^{27}$ Kuschel, N. and Rackwitz, R., "Two basic problems in reliability based structural optimization," Mathematical Methods of Operations Research, Vol. 46, 1997, pp. 309-333.

18 of 21 


\section{List of Figures}

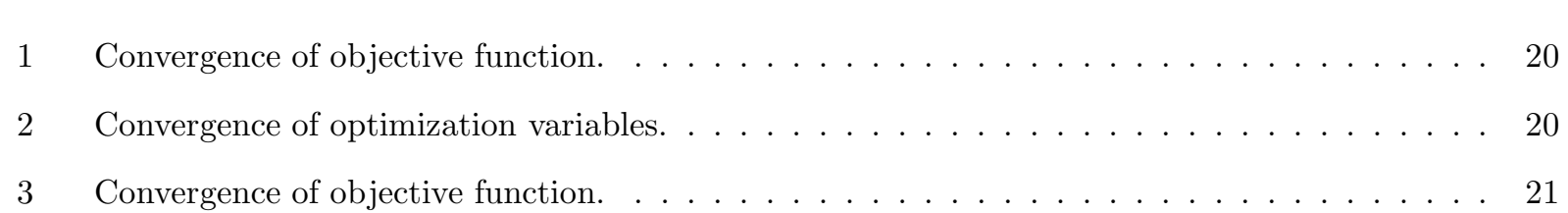

\section{List of Tables}

1 Stochastic Parameters in Short Column Desion Problem . . . . . . . . . . . . . . . . . 21

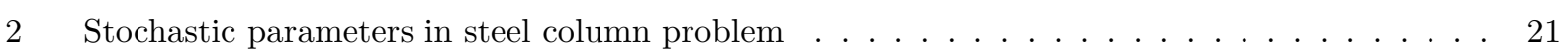




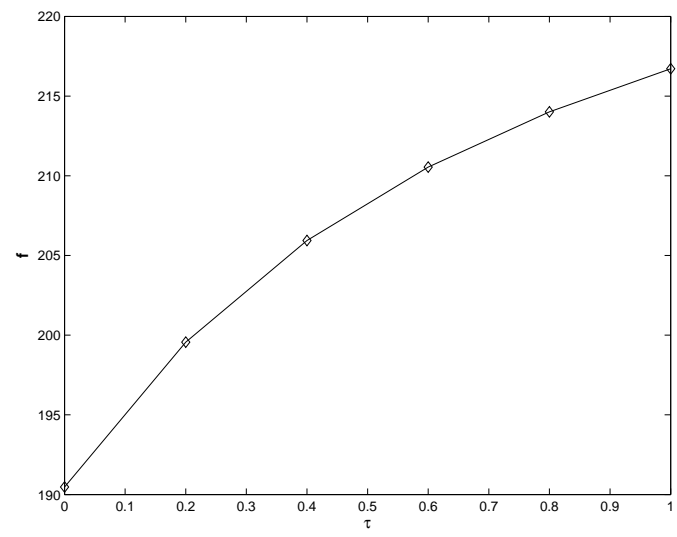

Figure 1. Convergence of objective function.
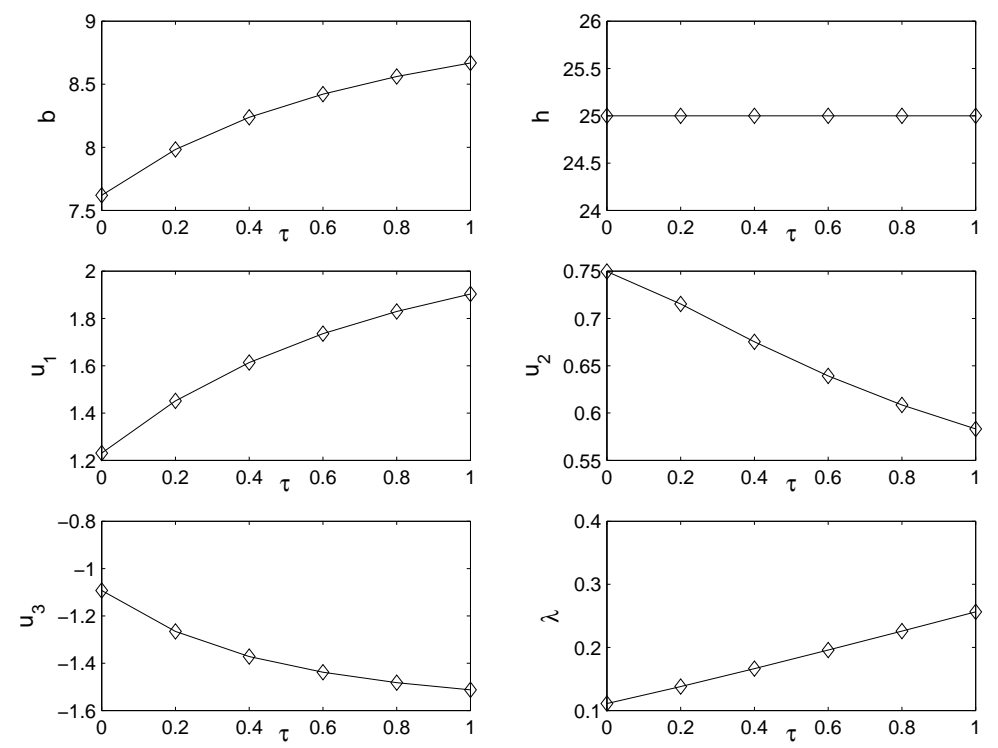

Figure 2. Convergence of optimization variables. 


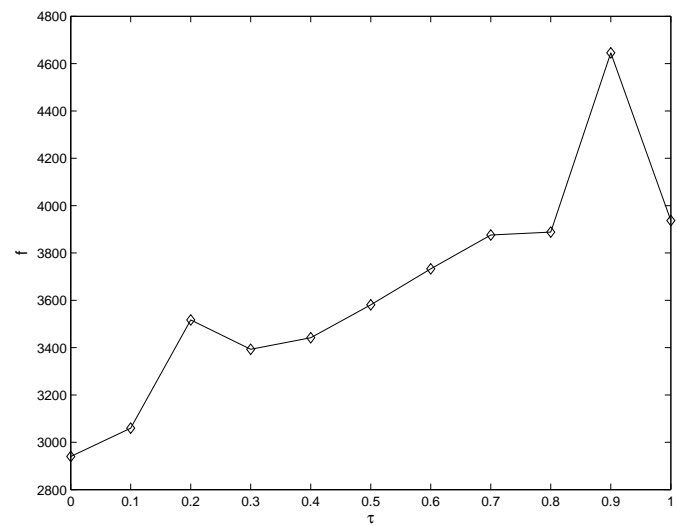

Figure 3. Convergence of objective function.

Table 1. Stochastic Parameters in Short Column Design Problem

\begin{tabular}{|c|c|c|c|c|c|c|c|}
\hline Variable & Symbol & Distribution & Mean/St. dev. & Unit & Corr. P & Corr. M & Corr. Y \\
\hline Yield Stress & $P$ & Normal & $500 / 100$ & $\mathrm{MPa}$ & 1 & 0.5 & 0 \\
\hline Bending Moments & $M$ & Normal & $2000 / 400$ & $\mathrm{MNm}$ & 0.5 & 1 & 0 \\
\hline Axial Force & $Y$ & Lognormal & $5 / 0.5$ & $\mathrm{MPa}$ & 0 & 0 & 1 \\
\hline
\end{tabular}

Table 2. Stochastic parameters in steel column problem

\begin{tabular}{|c|c|c|c|c|}
\hline Variable & Symbol & Distribution & Mean/Standard deviation & Unit \\
\hline Yield stress & $F_{s}$ & Lognormal & $400 / 35$ & $\mathrm{MPa}$ \\
\hline Dead weight load & $P_{1}$ & Normal & $500000 / 50000$ & $\mathrm{~N}$ \\
\hline Variable load & $P_{2}$ & Gumbel & $600000 / 90000$ & $\mathrm{~N}$ \\
\hline Variable load & $P_{3}$ & Gumbel & $600000 / 90000$ & $\mathrm{~N}$ \\
\hline Flange breadth & $B$ & Lognormal & $\mathrm{b} / 3$ & $\mathrm{~mm}$ \\
\hline Flange thickness & $D$ & Lognormal & $\mathrm{d} / 2$ & $\mathrm{~mm}$ \\
\hline Height of profile & $H$ & Lognormal & $\mathrm{h} / 5$ & $\mathrm{~mm}$ \\
\hline Initial Deflection & $F_{0}$ & Normal & $30 / 10$ & $\mathrm{~mm}$ \\
\hline Young's modulus & $E$ & Weibull & $21000 / 4200$ & $\mathrm{Mpa}$ \\
\hline
\end{tabular}

